\title{
热电材料中的高熵结构设计
}

\author{
蔡剑锋 ${ }^{1}$, 王泓翔 ${ }^{1,2}$, 刘国强 ${ }^{1,2}$, 蒋 俊 ${ }^{1,2}$ \\ (1. 中国科学院 宁波材料技术与工程研究所, 宁波 315201; 2. 中国科学院大学, 北京 100049)
}

摘 要: 高熵合金的设计思想在诸多材料领域都有广泛的应用, 本研究从高熵结构对热电输运性质的影响出发, 着 重讨论热电材料对高熵结构设计的一些要求。以 $\mathrm{CuInTe}_{2}$ 为实例, 提出了热电材料的高摘结构应当尽量减小晶格畸 变, 尽量选择在不影响费米面结构的格点位进行高摘掺杂。依据这些准则, 设计的高熵化合物 $\mathrm{Cu}_{0.8} \mathrm{Ag}_{0.2} \mathrm{Zn}_{0.1} \mathrm{Ga}_{0.4} \mathrm{Ge}_{0.1} \mathrm{In}_{0.4} \mathrm{Te}_{2}$ 的室温热导率降到了 $2.1 \mathrm{~W} \cdot \mathrm{m}^{-1} \cdot \mathrm{K}^{-1}$, 比基体材料降低 $70 \%$, 最高 $Z T$ 值达到 1.02, 较 基体提升 $90 \%$ 。在二元化合物 $\mathrm{SnTe}$ 中进行了 $\mathrm{AgSbSe}_{2}$ 固溶, 其室温热导率降到 $1.3 \mathrm{~W} \cdot \mathrm{m}^{-1} \cdot \mathrm{K}^{-1}$, 比基体降低 $80 \%$ 以 上。本研究表明, 遵循一定准则设计的高摘结构对于提升热电材料性能具有重要的意义。

关 键 词: 高熵合金; 热电材料; 吉布斯自由能; 高摘结构

中图分类号: TQ174 文献标识码: A

\section{Designing High Entropy Structure in Thermoelectrics}

\section{CAI Jianfeng ${ }^{1}$, WANG Hongxiang ${ }^{1,2}$, LIU Guoqiang ${ }^{1,2}$, JIANG Jun ${ }^{1,2}$}

(1. Ningbo Institute of Materials Technology and Engineering, Chinese Academy of Sciences, Ningbo 315201, China; 2. University of Chinese Academy of Sciences, Beijing 100049, China)

\begin{abstract}
With the fascinating properties observed in high entropy alloys, the idea of high entropy design has been applied to many material fields. Thermoelectric materials have some particular requirements for high entropy structure according to their transport characteristics. Here, we revealed that the high entropy structure for thermoelectrics required less lattice distortion, and the doping sites should have less influence on the Fermi surface. In the designed compound of $\mathrm{Cu}_{0.8} \mathrm{Ag}_{0.2} \mathrm{Zn}_{0.1} \mathrm{Ga}_{0.4} \mathrm{Ge}_{0.1} \mathrm{In}_{0.4} \mathrm{Te}_{2}$, the room-temperature thermal conductivity is reduced by $80 \%$ as compared to the matrix, and the maximum $Z T$ is enhanced to 1.02. In SnTe, the solid solution of AgSbSe $\mathrm{P}_{2}$ reduces the room-temperature thermal conductivity by $80 \%$, reaching $1.3 \mathrm{~W} \cdot \mathrm{m}^{-1} \cdot \mathrm{K}^{-1}$. This study shows that the high entropy structure following the proposed designing rules could be an important strategy for thermoelectrics.
\end{abstract}

Key words: high entropy alloys; thermoelectrics; Gibbs free energy; high entropy structure

高熵合金(High Entropy Alloys)概念最初来自金 属材料领域, 它是指由五种及五种以上组元形成的 合金体系, “高熵”反映的正是材料结构的无序性 ${ }^{[1-2]}$ 。
与传统的合金材料相比，高熵合金具有以下四方面 的显著特点：热力学高熵效应、晶格畸变效应、动 力学迟滞扩散效应和性能上的“鸡尾酒”效应, 这些

收稿日期：2020-11-18; 收到修改稿日期：2020-12-10; 网络出版日期：2020-12-21

基金项目: 宁波市科技创新 2025 重大专项(2019B10085)

Ningbo Science and Technology Innovation 2025 Major Project (2019B10085)

作者简介: 蔡剑锋(1995-), 男, 硕士研究生. E-mail: caijianfeng@nimte.ac.cn CAI Jianfeng(1995-), male, Master candidate. E-mail: caijianfeng@nimte.ac.cn

通信作者: 蒋 俊, 研究员. E-mail: jjun@nimte.ac.cn JIANG Jun, professor. E-mail: jjun@nimte.ac.cn 
效应使得高熵合金具有很多优异的性能 ${ }^{[3]}$, 如高强 度 $^{[4-5]}$ 、高硬度 ${ }^{[6]}$ 、耐磨 ${ }^{[7]}$ 、耐腐蚀 ${ }^{[8]}$ 、抗高温氧化 ${ }^{[9]}$ 、 抗高温软化 ${ }^{[10]}$ 等。近年来研究发现高熵结构不仅能 够提升材料的力学性能, 而且对材料很多物理性能 也有复杂的影响, 因此高熵合金的设计思路逐渐拓 展到磁性材料 ${ }^{[11-12]}$ 、介电材料 ${ }^{[13]}$ 和热电材料 ${ }^{[14-15]}$ 等领域。设计高熵结构除了要遵循一些共有的准则 外, 不同领域也有其特殊性, 这主要取决于对材料 性能的不同需求 ${ }^{[16-17]}$ 。

热电材料是一种能够直接将热能转换为电能的 功能材料, 热电器件在工作时无需传动部件, 具有 无噪音、结构简单、可靠性高等优点, 在废热回收、 微型制冷等领域有着广泛的应用前景 ${ }^{[18]}$ 。热电材料 的性能通常用热电优值 $Z T$ 来表征, $Z T=S \sigma T / \kappa$, 式中 $\boldsymbol{S}, \sigma, T$ 和 $\kappa$ 分别表示赛贝克系数、电导率、温度和 热导率, 其中热导率主要由电子和晶格热导率两部 分组成 ${ }^{[19]}$ 。从热电优值的表达式可以看出, 优质的 热电材料需要有高赛贝克系数、高电导率和低热导 率, 但由于上述参数在物理上耦合在一起, 要实现 高 $Z T$ 往往比较困难 ${ }^{[20]}$ 。近年来, 研究者发现高熵合 金的设计理念对热电材料研究也有着重要的应用。 本研究将从高摘结构对热电输运性质的影响出发, 探索热电材料中高熵结构设计的可能性。

\section{1 结构熵与热电输运参数}

合金体系的结构熵可以表示为:

$$
\Delta S=k_{\mathrm{B}} \ln \Omega=-N_{\mathrm{A}} k_{\mathrm{B}} \sum_{i=1}^{n} x_{i} \ln x_{i}
$$

其中, $k_{\mathrm{B}}$ 为玻尔兹曼常数, $\Omega$ 为许可的构型数目, $x_{i}$ 是第 $i$ 个组分的摩尔含量, $N_{\mathrm{A}}$ 为阿伏伽德罗常数。 从公式(1)可以看出, 体系的组元越多, 组元比例越 接近, 体系的结构熵就越高。

系统的固溶稳定性由吉布斯自由能 $(G)$ 决定, $G$
可由公式(2)计算得到:

$$
G=H-T S
$$

式中, $H$ 为焓, $T$ 为温度, $S$ 为熵。当系统掺杂后的吉 布斯自由能小于掺杂前的, 即可形成稳定的固溶 相。由公式(2)可以看出, 系统的熵越大, 吉布斯自 由能越小, 越有利于形成均一的固溶相。

对比高熵合金的四大效应，可以发现高熵结构 对热电材料性能的影响主要体现在热导率与电导率 上。高熵结构标志着材料体系有序度的下降, 会降 低体系的电导率和热导率。对于热电材料的性能而 言, 热导率的下降是有利的, 而电导率的下降是不 利的。通常认为高熵结构导致晶格热导率降低主要 源自晶格畸变效应, 晶格畸变会降低声子速度, 并 增加声子散射几率。然而根据声子输运的基本规律 分析可以发现, 高熵结构对热导率的降低是一种本 征行为, 声子频支的数目等于不等价原子数目的 3 倍, 声子频支的增加可以提高声子散射几率。同位 素掺杂会降低热导率, 就是由于声子频支的增加造 成的 ${ }^{[21]}$ 。对热电材料而言, 高熵结构一直有利于热 导率的降低, 所以在设计结构时应尽量避免晶格畸 变, 以减少对电导率的影响, 为此应选择原子半径 和价态与基体元素接近的固溶元素。

相对热导率与电导率而言, 结构熵对赛贝克系 数的影响较为复杂。因为塞贝克系数与费米面能谷 的简并度呈正相关依赖关系, 高熵体系倾向于形成 密排的高对称性结构, 因此有观点认为高熵结构有 利于提升赛贝克系数。但是这种观点有一定的局限 性, 高对称性晶体结构并不一定会提高费米面能谷 的简并度。以类金刚石结构的 $\mathrm{CuInTe}_{2}$ 为例, 其晶体 结构与能带结构如图 1 所示。类金刚石结构具有较 高的晶体对称性, 但其价带顶和导带底均位于倒空 间中心的 Gamma 点上, 能谷简并度为 1 。这表明晶 体对称性与能谷对称性不是简单的一一对应关系, 晶体对称性的提高并不一定会提升塞贝克系数。另 (a)

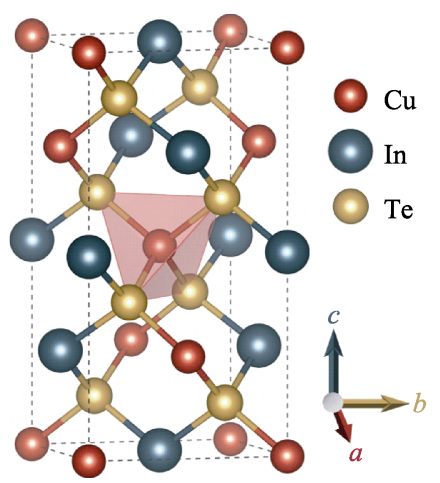

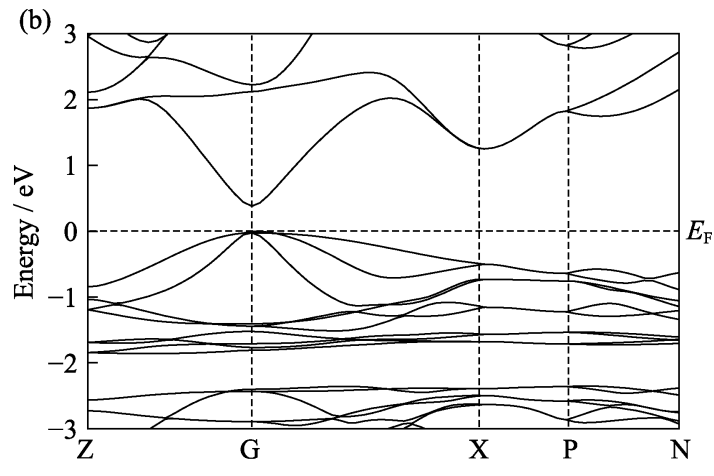

图 $1 \mathrm{CuInTe}_{2}$ 的晶体结构(a)与电子能带结构(b)

Fig. 1 Crystal structure (a) and electronic structure (b) of CuInTe 
外, 如果掺杂元素在能隙内引入新的能带, 能隙的 减小反而会降低塞贝克系数。如果基体材料本身就 具有较好的功率因子, 在设计高熵结构时, 应尽量 避免掺杂元素对带边结构产生影响。下面仍以 $\mathrm{CuInTe}_{2}$ 为例解释这一准则。

图 2 是采用第一性原理计算得到的 CuInTe 2 电 子能态密度, 以及 $\mathrm{Cu}-\mathrm{d}$ 和 Te-p 的分波态密度。从 价态分析即可知道, CuInTe 2 的价带边主要由 $\mathrm{Cu}-\mathrm{d}$ 轨道贡献, 而导带边主要由 Te-p 轨道贡献, 从图 2 可以清楚地看出这点。相应的, In 的电子轨道对带 边的贡献较小, 所以在对 $\mathrm{CuInTe}_{2}$ 类金刚石结构进 行高熵设计时, 应尽量选择在 In 格点位进行掺杂。

\section{2 三元化合物 $\mathrm{CuInTe}_{2}$ 的高熵结构设计}

铜基类金刚石材料, 包括 $\mathrm{CuInTe}_{2}{ }^{[22]}$, $\mathrm{CuGaTe}_{2}{ }^{[23]}, \mathrm{Cu}_{2} \mathrm{SnS}_{3}{ }^{[24]}, \mathrm{Cu}_{3} \mathrm{SbSe}_{4}{ }^{[25]}$ 等, 是一类重 要的热电材料体系。这类材料具有较高的晶体对称 性、高热稳定性和优良的机械性能等, 但其晶格热 导率普遍较高。如何降低热导率成为铜基热电材料 研究的核心问题之一, 研究者采用了不同的策略对 其进行调控, 熵工程是其中最有效的方法。Liu 等 ${ }^{[26]}$ 在研究的高熵化合物 $\mathrm{Cu}_{0.5} \mathrm{Ag}_{0.5} \mathrm{In}_{0.5} \mathrm{Ga}_{0.5} \mathrm{Te}_{2}$ 中获得 了 $1.8 \mathrm{~W} \cdot \mathrm{m}^{-1} \cdot \mathrm{K}^{-1}$ 的室温晶格热导率, 比基体 $\mathrm{CuInTe}_{2}$ 的晶格热导率降低了 $80 \%$ 左右, 他们对两 个阳离子格位进行了等同的高熵掺杂。依据前述讨 论, 在 In 离子位进行掺杂应更有利于提升热电性能, 本研究将通过实验进一步澄清此问题。

首先制备 CuInTe 2 和 $\mathrm{Cu}_{0.5} \mathrm{Ag}_{0.5} \mathrm{In}_{0.5} \mathrm{Ga}_{0.5} \mathrm{Te}_{2}$, 然 后在 In 位进行固溶。Ga 是 In 同族近邻元素, 具有 相似的化学性质和接近的原子尺寸, 是一个较好的 固溶元素。Zn 和 Ge在元素周期表中也与 In近邻, 同 时选择这两种元素是为了保持价态平衡。为了提高

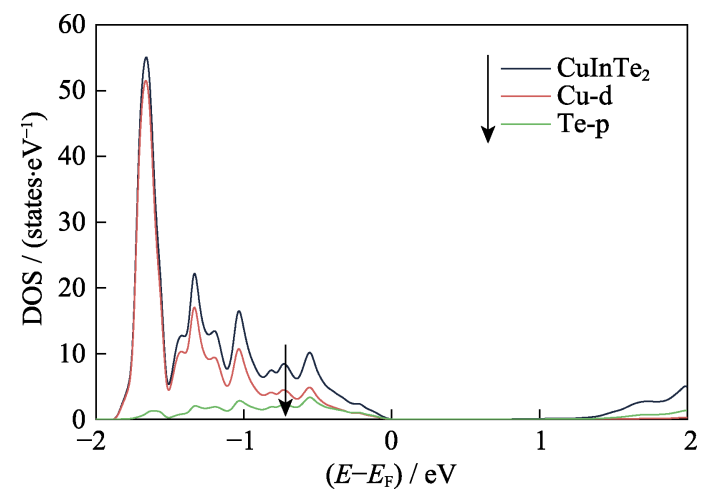

图 $2 \mathrm{CuInTe}_{2}$ 的电子能态密度, 包括 $\mathrm{Cu}-\mathrm{d}$ 与 $\mathrm{Te}-\mathrm{p}$ 分波态密度 Fig. 2 Density of State (DOS) of $\mathrm{CuInTe}_{2}$, including the partial DOS for $\mathrm{Cu}-\mathrm{d}$ and Te-p orbitals
结构熵, 在 $\mathrm{Cu}$ 位和 $\mathrm{In}$ 位都进行等摩尔比固溶, 设计 组分为 $\mathrm{Cu}_{0.5} \mathrm{Ag}_{0.5} \mathrm{Zn}_{0.25} \mathrm{Ga}_{0.25} \mathrm{Ge}_{0.25} \mathrm{In}_{0.25} \mathrm{Te}_{2}$ 。依据前 述准则, 为减小对电导率的影响又设计了一个新的 组分 $\mathrm{Cu}_{0.8} \mathrm{Ag}_{0.2} \mathrm{Zn}_{0.1} \mathrm{Ga}_{0.4} \mathrm{Ge}_{0.1} \mathrm{In}_{0.4} \mathrm{Te}_{2}$ 。采用熔融热压 工艺制备了上述 4 个样品, 图 3 为四个样品的 $\mathrm{X}$ 射 线衍射(XRD)图谱, 从图中可以看出, 这四个样品 的 XRD 图谱均与标准卡片吻合, 没有出现明显的 杂质峰, 表明制备的样品都是质量较好的单一固溶 相。图中各个样品的衍射峰产生了不同程度偏移, 这 是由固溶元素与基体元素的原子半径差异造成的。

图 4 是四个样品测量得到的电导率和塞贝克系

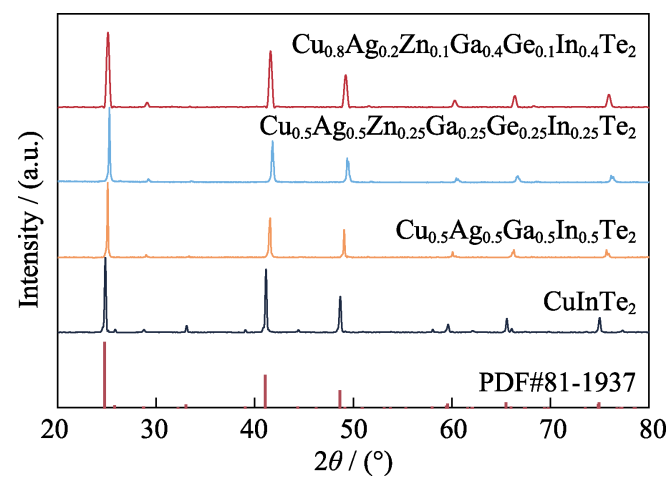

图 $3 \mathrm{CuInTe}_{2}$ 及其高熵固溶化合物的 XRD 图谱

Fig. 3 XRD patterns for CuInTe $e_{2}$ and its high entropy solid solutions
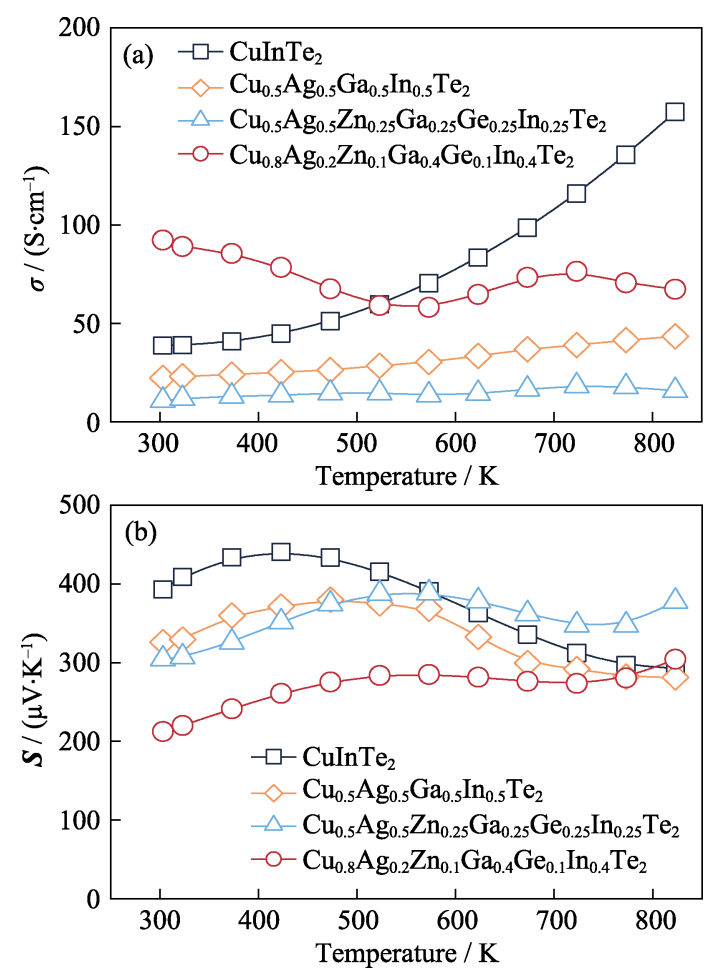

图 $4 \mathrm{CuInTe}_{2}$ 及其高熵固溶化合物的电导率(a)及塞贝克系 数(b)

Fig. 4 Electrical conductivity (a) and Seebeck coefficient (b) for $\mathrm{CuInTe}_{2}$ and its high entropy solid solutions 
数。样 品 $\mathrm{Cu}_{0.5} \mathrm{Ag}_{0.5} \mathrm{In}_{0.5} \mathrm{Ga}_{0.5} \mathrm{Te}_{2}$ 和 $\mathrm{Cu}_{0.5} \mathrm{Ag}_{0.5} \mathrm{Zn}_{0.25} \mathrm{Ga}_{0.25} \mathrm{Ge}_{0.25} \mathrm{In}_{0.25} \mathrm{Te}_{2}$ 的电导率在整个 温区的电导率都低于基体 $\mathrm{CuInTe}_{2}$, 具有最大结构 熵的样品 $\mathrm{Cu}_{0.5} \mathrm{Ag}_{0.5} \mathrm{Zn}_{0.25} \mathrm{Ga}_{0.25} \mathrm{Ge}_{0.25} \mathrm{In}_{0.25} \mathrm{Te}_{2}$ 的电导 率最低。掺杂样品的赛贝克系数相较基体也有一定 变化, 主要源自载流子浓度的变动, 如表 1 所示。

图 5 是四个样品测量得到的热导率以及最终的 热电优值 $Z T$ 。样品 $\mathrm{Cu}_{0.5} \mathrm{Ag}_{0.5} \mathrm{In}_{0.5} \mathrm{Ga}_{0.5} \mathrm{Te}_{2}$ 的室温热 导率约为 $2.5 \mathrm{~W} \cdot \mathrm{m}^{-1} \cdot \mathrm{K}^{-1}$, 比基体 $\left(6.7 \mathrm{~W} \cdot \mathrm{m}^{-1} \cdot \mathrm{K}^{-1}\right)$ 明 显减小，但高于之前的报道数值。样品 $\mathrm{Cu}_{0.5} \mathrm{Ag}_{0.5} \mathrm{Zn}_{0.25} \mathrm{Ga}_{0.25} \mathrm{Ge}_{0.25} \mathrm{In}_{0.25} \mathrm{Te}_{2}$ 的室温热导率进 一步降低到 $1.8 \mathrm{~W} \cdot \mathrm{m}^{-1} \cdot \mathrm{K}^{-1}$, 并且它的热导率在整个 温区都显著低于 $\mathrm{Cu}_{0.5} \mathrm{Ag}_{0.5} \mathrm{In}_{0.5} \mathrm{Ga}_{0.5} \mathrm{Te}_{2}$, 这表明高熵 结构越复杂, 其热导率越低。对于组分优化后的样 品 $\mathrm{Cu}_{0.8} \mathrm{Ag}_{0.2} \mathrm{Zn}_{0.1} \mathrm{Ga}_{0.4} \mathrm{Ge}_{0.1} \mathrm{In}_{0.4} \mathrm{Te}_{2}$, 其室温热导率略 有提升, 约为 $2.1 \mathrm{~W} \cdot \mathrm{m}^{-1} \cdot \mathrm{K}^{-1}$ 。

表 1 室温下不同组分样品的载流子浓度与迁移率

Table 1 Carrier concentrations and mobilities of samples with different components at room temperature

\begin{tabular}{ccc}
\hline Sample & $n_{\mathrm{H}} / \mathrm{cm}^{-3}$ & $\begin{array}{c}\mu_{\mathrm{H}} / \\
\left(\mathrm{cm}^{2} \cdot \mathrm{V}^{-1} \cdot \mathrm{s}^{-1}\right)\end{array}$ \\
\hline CuInTe & $3.1 \times 10^{18}$ & 78.1 \\
$\mathrm{Cu}_{0.5} \mathrm{Ag}_{0.5} \mathrm{Ga}_{0.5} \mathrm{In}_{0.5} \mathrm{Te}_{2}$ & $1.3 \times 10^{18}$ & 10.4 \\
$\mathrm{Cu}_{0.5} \mathrm{Ag}_{0.5} \mathrm{Zn}_{0.25} \mathrm{Ga}_{0.25} \mathrm{Ge}_{0.25} \mathrm{In}_{0.25} \mathrm{Te}_{2}$ & $5.6 \times 10^{19}$ & 1.2 \\
$\mathrm{Cu}_{0.8} \mathrm{Ag}_{0.2} \mathrm{Zn}_{0.1} \mathrm{Ga}_{0.4} \mathrm{Ge}_{0.1} \mathrm{In}_{0.4} \mathrm{Te}_{2}$ & $3.2 \times 10^{19}$ & 17.8 \\
\hline
\end{tabular}
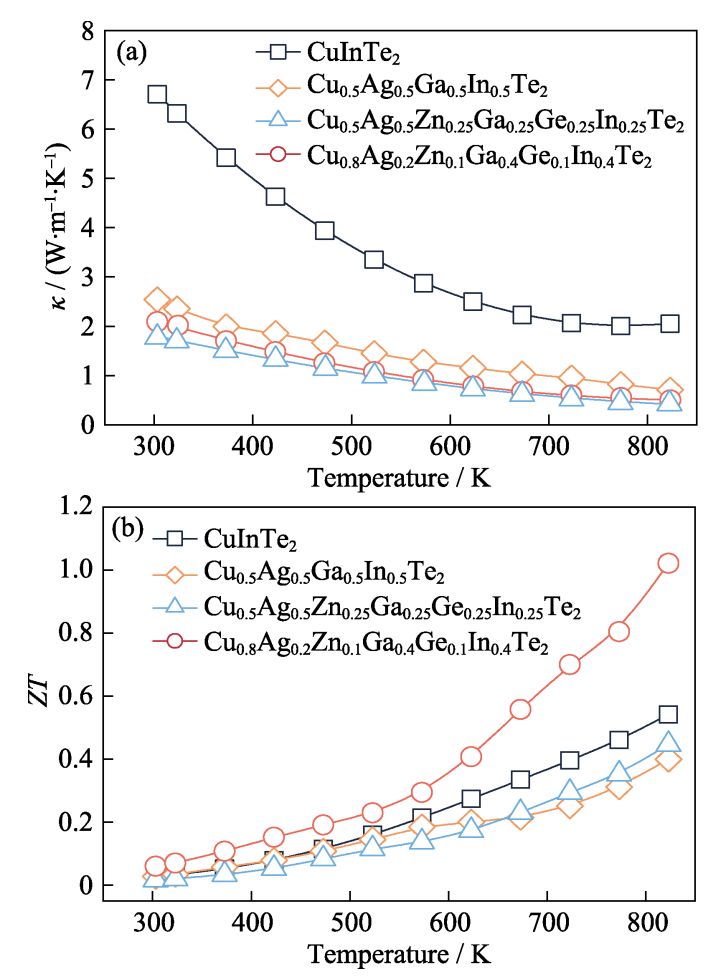

图 $5 \mathrm{CuInTe}_{2}$ 及其高熵固溶化合物的热导率(a)及热电优值(b) Fig. 5 Thermal conductivity (a) and $Z T$ (b) for CuInTe $\mathrm{C}_{2}$ and its high entropy solid solutions
表 1 列出了四个样品的载流子浓度与迁移率。 基体具有较高的迁移率, 达到 $78.1 \mathrm{~cm}^{2} /(\mathrm{V} \cdot \mathrm{s})$, $\mathrm{Cu}_{0.5} \mathrm{Ag}_{0.5} \mathrm{In}_{0.5} \mathrm{Ga}_{0.5} \mathrm{Te}_{2}$ 的迁移率降低到 $10.4 \mathrm{~cm} /(\mathrm{V} \cdot \mathrm{s})$ 熵最高的 $\mathrm{Cu}_{0.5} \mathrm{Ag}_{0.5} \mathrm{Zn}_{0.25} \mathrm{Ga}_{0.25} \mathrm{Ge}_{0.25} \mathrm{In}_{0.25} \mathrm{Te}_{2}$ 迁移率 急剧降低到 $1.2 \mathrm{~cm}^{2} /(\mathrm{V} \cdot \mathrm{s})$ 。依据前述讨论, 迁移率的 降低主要归因于晶格畸变和在 $\mathrm{Cu}$ 格点位的 $\mathrm{Ag}$ 固 溶。 $\mathrm{Zn}$ 和 $\mathrm{Ge}$ 虽然是 In 的近邻元素, 但不是同族元 素, 化学性质和原子尺寸与 In 的差异较大, 所以本 研究适当降低了 $\mathrm{Zn}$ 、 Ge 以及 $\mathrm{Ag}$ 的固溶比例。样品 $\mathrm{Cu}_{0.8} \mathrm{Ag}_{0.2} \mathrm{Zn}_{0.1} \mathrm{Ga}_{0.4} \mathrm{Ge}_{0.1} \mathrm{In}_{0.4} \mathrm{Te}_{2}$ 的迁移率又提升到 $17.8 \mathrm{~cm}^{2} /(\mathrm{V} \cdot \mathrm{s})$, 这证实了本研究组分设计的合理 性。随着迁移率的提升, 高熵结构设计样品的最高 $Z T$ 值达到 1.02@820K, 比基体提升了 100\%, 如 图 5(b)所示。虽然 $\mathrm{Cu}_{0.5} \mathrm{Ag}_{0.5} \mathrm{Zn}_{0.25} \mathrm{Ga}_{0.25} \mathrm{Ge}_{0.25} \mathrm{In}_{0.25} \mathrm{Te}_{2}$ 具有最低的热导率, 但其最高 $Z T$ 值仅为 0.4 , 甚至 低于基体, 这也表明热电材料不能仅仅追求高结构 熵, 其组分设计还应充分考虑热电输运的特点。

\section{3 二元化合物 SnTe 的高熵结构设计}

在三元化合物 $\mathrm{CuInTe}_{2}$ 中, 前述分析认为应尽 量在 In 位进行固溶掺杂, 因为 In 对带边电子结构的 影响远小于 $\mathrm{Cu}$ 和 $\mathrm{Te}$ 。对于三元及以上化合物, 比 较容易找到具有此种特点的格位。但是对于二元化 合物, 在两类格点的固溶一定会影响带边电子结构, 改变赛贝克系数并降低载流子迁移率, 因而其高熵 结构设计就更为复杂。以二元化合物 SnTe 为例, 在 其高熵固溶体 $\left(\mathrm{Sn}_{0.74} \mathrm{Ge}_{0.2} \mathrm{~Pb}_{0.1}\right)_{0.75} \mathrm{Mn}_{0.275} \mathrm{Te}$ 中, 研究 者观测到极低的晶格热导率 $\left(0.34 \mathrm{~W} \cdot \mathrm{m}^{-1} \cdot \mathrm{K}^{-1}\right)$ 以及 较高的热电优值, 其复杂组分的设计正是为了兼顾 电学性能 ${ }^{[27]}$ 。

对于 SnTe, 研究者发现对其进行 $\mathrm{AgSbTe}_{2}$ 和 $\mathrm{AgSbSe}_{2}$ 固溶, 同样可以获得很低的晶格热导率 ${ }^{[28-29]}$ 。 在 $\mathrm{SnTe}-\mathrm{AgSbSe}_{2}$ 固溶体中测量得到的热导率以及 电导率, 如图 6 所示 ${ }^{[28]}$, 从图中可以发现固溶体相 较基体, 电导率与热导率都有非常显著的降低, 因 而对热电优值的影响取决于两个因素的竞争。在高 温区, 固溶体热导率的下降幅度大于电导率的下降 幅度, 因此其高温 $Z T$ 值比基体有一定提升。这表明 在 $\mathrm{Sn}$ 位进行 $\mathrm{Ag}$ 和 $\mathrm{Sb}$ 固溶有利于提升热电性能, 原 因仍然是这两种固溶元素对带边电子结构的影响相 对较小。SnTe 的带边结构由 Sn-p 和 Te-p 轨道贡献, 而参与成键的 Ag-d 轨道和 Sb-p 轨道的能级分别为 -19.2 和 $-8.4 \mathrm{eV}$, 均明显小于 $\mathrm{Sn}-\mathrm{p}$ 轨道能级 $(-6.8 \mathrm{eV})^{[30]}$ 。从化学键分析可知, 这两种固溶元素 产生的能态密度不会在带隙边出现峰值, 因而对电 

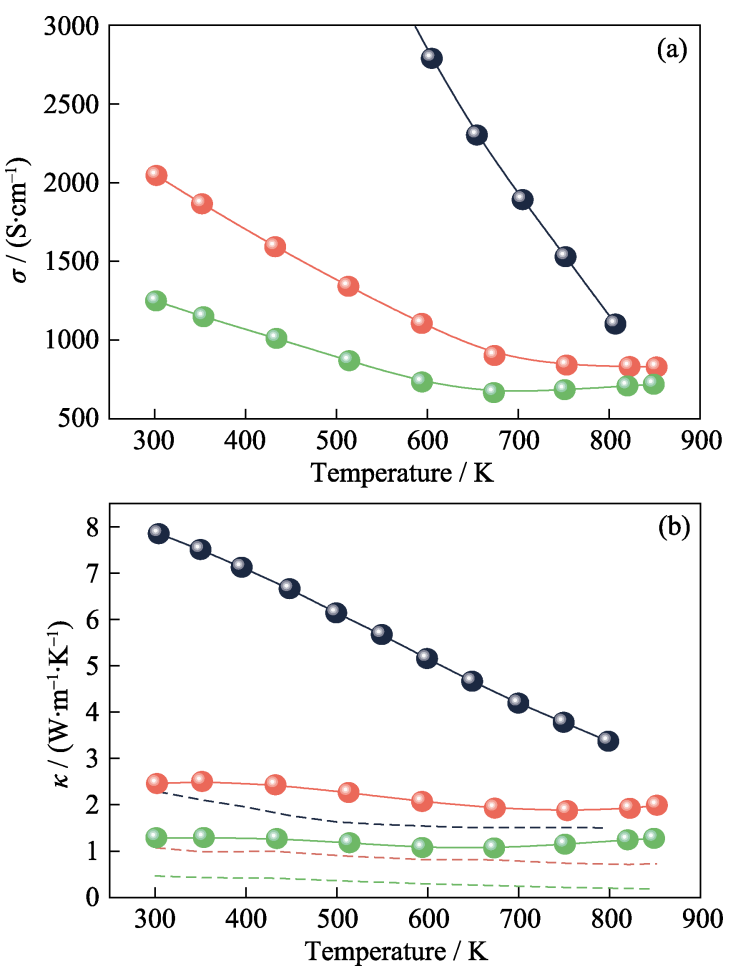

图 6 SnTe-AgSbSe 2 固溶体的电导率(a)及热导率(b) ${ }^{[28]}$

Fig. 6 Electrical conductivity (a) and thermal conductivity (b) for $\mathrm{SnTe}-\mathrm{AgSbSe}_{2}$ solid solutions ${ }^{[28]}$

Solid lines refer to total thermal conductivity, dash lines refer to lattice thermal conductivity. and black, red and green refer to SnTe, SnTe+ $10 \% \mathrm{AgSbSe}_{2}$ and $\mathrm{SnTe}^{2} 20 \% \mathrm{AgSbSe}_{2}$, respectively

导率的影响相对较小。这个例子表明对二元化合物 的高熵结构设计更为复杂, 固溶元素不能简单地选 取元素周期表上的近邻元素, 还需要考虑不同元素 的电子能级分布。

\section{4 结束语}

高熵合金的设计思路已经对很多材料领域产生 重要的影响, 高结构熵可以提升体系的固溶度极限, 有利于形成均一的稳定相, 这点对热电材料有着重 要意义。高熵结构标志着体系有序度的下降, 导致 材料电导率和热导率同时下降，这是热电材料高 熵结构设计的主要矛盾。通过理论分析, 本研究提 出了热电材料高熵结构设计需要注意的两点：一 是通过固溶元素选择尽量减少晶格畸变; 二是尽 量选择在不影响电子费米面结构的格位进行固溶 掺杂。遵循这两点要求, 本研究设计了 $\mathrm{CuInTe}_{2}$ 的 高熵结构, 在极大降低系统热导率的同时, 尽量减 小对载流子迁移率的影响, 将材料的热电优值提升 了 $120 \%$ 。

本文着重讨论了结构熵对热导率及电导率的影 响, 并未就其对赛贝克系数的影响展开讨论。熵是
描述热力学系统的基本物理量, 赛贝克系数原则上 也可以用熵来描述，但其直接对应的是电子熵，而 非晶格的结构熵。由于这一原因, 结构熵对赛贝克 系数的影响非常复杂, 本研究提出的高熵结构设计 准则也是尽量减少其对赛贝克系数的影响。如果能 将电子熵与结构熵结合起来, 发展出更基础的高熵 理论, 将能极大促进热电材料的研究发展。

\section{参考文献:}

[1] GEORGEE P, RAABE D, RITCHIER O. High-entropy alloys. Nature Reviews Materials, 2019, 4(8): 515-534.

[2] MIRACLED B, SENKOVO N. A critical review of high entropy alloys and related concepts. Acta Mater., 2017, 122: 448-511.

[3] YEH J W, CHEN S K, LIN S J, et al. Nanostructured high-entropy alloying with multiple principal elements: novel alloy design concepts and outcomes. Adv. Eng. Mater., 2004, 6(5): 299-303.

[4] GLUDOVATZ B, HOHENWARTER A, CATOOR D, et al. A fracture-resistant high-entropy alloy for cryogenic applications. Science, 2014, 345(6201): 1153-1158.

[5] LI Z, PRADEEPK G, DENG Y, et al. Metastable high-entropy dual-phase alloys overcome the strength-ductility trade-off. Nature, 2016, 534(7606): 306-307.

[6] YOUSSEF K M, ZADDACH A J, NIU C, et al. A novel low-density, high-hardness, high-entropy alloy with close-packed single-phase nanocrystalline structures. Materials Research Letters, 2014, 3(2): 95-99.

[7] ZHANG Y, ZUO T T, TANG Z, et al. Microstructures and properties of high-entropy alloys. Prog. Mater. Sci., 2014, 61: $1-93$.

[8] SHI Y Z, YANG B, LIAW P. Corrosion-resistant high-entropy alloys: a review. Metals-Basel, 2017, 7(2): 43-1-18.

[9] SENKOV O N, WILKS G B, MiRACle D B, et al. Refractory high-entropy alloys. Intermetallics, 2010, 18(9): 1758-1765.

[10] HSU C Y, JUAN C C, WANG W R, et al. On the superior hot hardness and softening resistance of $\mathrm{AlCoCr}_{x} \mathrm{FeMo}_{0.5} \mathrm{Ni}$ highentropy alloys. Materials Science and Engineering: A, 2011, 528(10/11): 3581-3588.

[11] OIKAWA K, ITO W, IMANO Y, et al. Effect of magnetic field on martensitic transition of $\mathrm{Ni}_{46} \mathrm{Mn}_{41} \mathrm{In}_{13}$ heusler alloy. Appl. Phys. Lett., 2006, 88(12): 122507-1-3.

[12] ZHANG Y, ZUO T, CHENG Y, et al. High-entropy alloys with high saturation magnetization, electrical resistivity, and malleability. Sci. Rep., 2013, 3: 1455-1-7.

[13] BÉRARDAN D, FRANGER S, DRAGOE D, et al. Colossal dielectric constant in high entropy oxides. Physica Status Solidi-Rapid Research Letters, 2016, 10(4): 328-333.

[14] SHAFEIE S, GUO S, HU Q, et al. High-entropy alloys as hightemperature thermoelectric materials. J. Appl. Phys., 2015, 118(18): 184905-1-10.

[15] WEI P C, LIAO C N, WU H J, et al. Thermodynamic routes to ultralow thermal conductivity and high thermoelectric performance. Adv. Mater., 2020, 32(12): 1906457-1-10.

[16] TSAI M H. Three strategies for the design of advanced highentropy alloys. Entropy, 2016, 18(7): 252-1-14.

[17] TSAI M H, YEH J W. High-entropy alloys: a critical review. Materials Research Letters, 2014, 2(3): 107-123.

[18] BELL L E. Cooling, heating, generating power, and recovering 
waste heat with thermoelectric systems. Science, 2008, 321(12): 1457-1461.

[19] SNYDER G J. Complex strucure thermoelectric meterial. Nat. Mater., 2008, 7(2): 105-114.

[20] SOOTSMAN J R, CHUNG D Y, KANATZIDIS M G. New and old concepts in thermoelectric materials. Angewandte Chemie International Edition, 2009, 48(46): 8616-8639.

[21] ZHANG H, LEE G, FONSECA A F, et al. Isotope effect on the thermal conductivity of graphene. Journal of Nanomaterials, 2010, 2010: 537657-1-5.

[22] LIU R, XI L, LIU H, et al. Ternary compound CuInTe 2 : a promising thermoelectric material with diamond-like structure. Chem. Commun., 2012, 48(32): 3818-3820.

[23] PLIRDPRING T, KUROSAKI K, KOSUGA A, et al. Chalcopyrite $\mathrm{CuGaTe}_{2}$ : a high-efficiency bulk thermoelectric material. $A d v$. Mater., 2012, 24(27): 3622-3626.

[24] XI L, ZHANGY B, SHIX Y, et al. Chemical bonding, conductive network, and thermoelectric performance of the ternary semiconductors $\mathrm{Cu}_{2} \mathrm{SnX}_{3}(\mathrm{X}=\mathrm{Se}, \mathrm{S})$ from first principles. Phys. Rev. B, 2012, 86(15): 155201-155215.
[25] SKOUGE J, CAINJ D, MORELLID T. High thermoelectric figure of merit in the $\mathrm{Cu}_{3} \mathrm{SbSe}_{4}-\mathrm{Cu}_{3} \mathrm{SbS}_{4}$ solid solution. Appl. Phys. Lett., 2011, 98(26): 261911-1-3.

[26] LIU R, CHEN H, ZHAO K, et al. Entropy as a gene-like performance indicator promoting thermoelectric materials. Adv. Mater., 2017, 29(38): 1702712-1-7.

[27] HU L, ZHANG Y, WU H, et al. Entropy engineering of SnTe: multi-principal-element alloying leading to ultralow lattice thermal conductivity and state-of-the-art thermoelectric performance. $A d v$. Energy Mater., 2018, 8(29): 1802116-1-14.

[28] LIN S X, TAN X J, SHAO H Z, et al. Ultralow lattice thermal conductivity in SnTe by manipulating the electron-phonon coupling. The Journal of Physical Chemistry C, 2019, 123(26): 15996-16002.

[29] TAN G, HAO S, HANUS R, et al. High thermoelectric performance in $\mathrm{SnTe}-\mathrm{AgSbTe}_{2}$ alloys from lattice softening, giant phonon-vacancy scattering, and valence band convergence. ACS. Energy Lett., 2018, 3(3): 705-712.

[30] HARRISON W. Elementary Electronic Structure. London: World Scientific Publishing Company, 2004. 